\title{
Modeling of Long-term Operating Regimes of Hydro Power Plants as Part of Energy and Water Systems in the Context of Uncertainty
}

\author{
Viacheslav Nikitin*, Nikolay Abasov, Evgeny Osipchuk \\ Melentiev Energy Systems Institute Siberian Branch of the Russian Academy of Sciences (ESI SB RAS), 134 Lermontov St., Irkutsk, \\ 664033, Russia
}

\begin{abstract}
Energy and water management systems are closely interrelated through the operating regimes of Hydro Power Plants (HPP) or HPP cascades. This is first of all, characteristic of energy systems with a large share of HPP. One of such systems is the energy system of Siberia, which includes the Angara-Yenisei cascade of HPP, the largest in Russia and one of the largest in the world. The studies were carried out on the example of this energy system and the water management system of the Angara and Yenisei basins. A specific feature of the energy and water management systems of Siberia is a significant impact of stochastic factors on their operation. These factors include natural (water inflow in the reservoirs in the spring-summer period and outdoor temperature in the autumn-winter period), and also economic (demand for electric and thermal energy, electricity and heat prices) factors. The paper presents mathematical models for the joint study of energy and water systems. These models factor in the specifics of the systems during planning for a period of up to 1 year. Modeling of the interconnected operation of energy and water systems makes it possible to solve important problems. These are the improvement in the reliability and stability of the considered systems, increase in their economic efficiency (minimization of electricity prices for consumers), assessment and minimization of various risks, prevention from or reduction in possible damages, rational planning of repairs, the formation of fuel reserves at thermal power plants, and others.
\end{abstract}

\section{Introduction}

Energy and water systems are managed by various departments, as a rule, independently of each other. The legal and regulatory framework for these systems in the Russian Federation works also separately. Planning the operation of these systems focuses primarily on normal (average) operating conditions. Practical problems of studying the HPP operating regimes, however, are related to the periods of extreme conditions and the effect of these conditions on the stability and reliability of the systems. The extreme climatic situations in the water-energy systems lead to negative consequences, including significant socio-economic damages. Therefore, it is important to timely make management decisions to prevent (mitigate) the consequences of such events. By way of illustration, note the situation in the Angara basin and the Siberian energy system in recent years. In 2014-2018, an extreme water shortage was observed in the reservoirs of the Angara cascade. During this period, electricity generation from HPP decreased by 15-20\%. All participants in the water management sector encountered problems, the overall reliability and stability of the water and energy systems significantly declined. The problem is compounded by the fact that the existing planning and management system in the electric power industry suggests the estimation of longterm energy system operating conditions and balances of electricity and power for a period of up to 1-5 years, while in water management, the forecasting horizon for water inflow into reservoirs and planning of HPP operating regimes is $1-3$ months, and the forecasting period for air temperature is limited to 5-10 days.

The problem of modeling the operation of energy systems with HPP, including optimization of their operation, started evolving as soon as energy systems came into existence. Modeling methods developed alongside with the complication of the systems and the emergence of new mathematical and information technologies. A great number of effective algorithms, methods and models have been developed in recent years to study the operating regimes of HPP and the functioning of energy and water systems. Many of them were created in the 1960s and 1970s and were subsequently used in automated dispatch control systems for energy systems, to perform water-energy calculations, and manage water and energy systems. Simulation and mathematical methods found wide use in the optimization of operating conditions (linear, nonlinear, dynamic programming, including stochastic and multi-criteria optimization, etc.) [1-5]. Apart from Russia and USA, there is a great experience in modeling

\footnotetext{
* Corresponding author: nikitin1310@mail.ru
} 
energy systems with high penetration of hydro in Norway, Canada, Brazil, and China [6-13].

Thus, to date, considerable experience has been gained in the practice of modeling energy systems with hydraulic and thermal power plants. Many issues, however, remain unsolved due to the complexity and versatility of the problem to be solved. Most models do not fully represent the constraints for hydroelectric power plants, such as the lack of data on the future hydrological regime of a probabilistic nature. This, in turn, leads to insufficient consideration of random nature-induced factors in planning the operating conditions of energy and water systems and ensuring their flexibility and reliability. This is especially true for long-term estimates of possible water inflows into HPP reservoirs and temperature changes.

Most modern methods and models for managing the long-term operating conditions of HPP, water and energy systems for a period of more than 3 months rely mainly on statistical information on long-term average changes in water inflows, and long-term average annual, monthly, summer and winter temperatures over past periods. Traditionally, the Monte Carlo method is most often used to generate forecast estimates of stochastic indicators. Some studies suggest the use of an integrated approach for managing cascades of reservoirs with the priorities of meeting either water system requirements or energy system requirements [14-16]. Joint long-term operation of the two systems (energy and water), as a rule, is not considered. This is especially true for climate factors.
Significant changes in socio-economic conditions, legislation, and regional climate changes in recent years associated with global processes require appropriate changes and additions to the methods and models of managing energy and water systems and their operation. The use of various prognostic methods and models is necessary to beforehand take account of possible changes in the indices of water content and temperature condition, including the data obtained with global climate models on the dynamics of the state of the atmosphere for a period of up to 1 year. Regular monitoring of such estimates and their refinement (at least 1 time per month) makes it possible to obtain more informed probabilistic scenarios of expected changes in natural factors for long-term planning of the energy and water systems. Their use allows increasing the efficiency of planning and management.

This paper presents an approach used at the Melentiev Energy Systems Institute SB RAS (ESI SB RAS) to jointly model long-term operating regimes of HPP and HPP cascades (for example, the AngaraYenisei cascade) within energy and water systems, given climatic and other factors.

\section{A system of models for the joint study of water and energy systems}

A research team of ESI SB RAS has developed an approach [17-18] to comprehensively study the longterm operation of water and energy systems with a large share of HPP (Fig. 1).

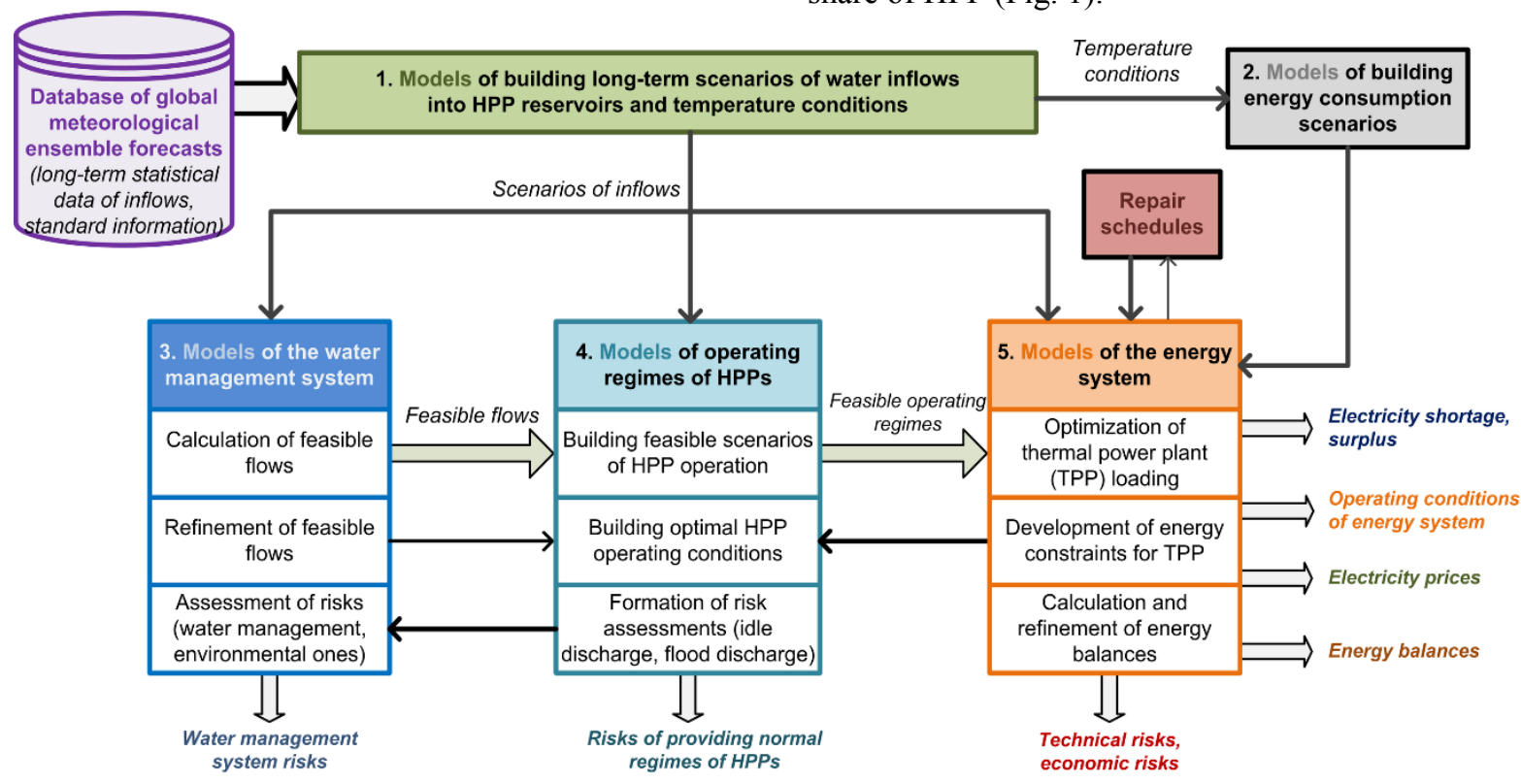

Fig. 1. The scheme of models for integrated studies of long-term operation of water and energy systems

This approach is implemented by 5 main blocks of the models:

1. Models of building long-term scenarios of water inflows into HPP reservoirs and temperature conditions in winter and summer in a probabilistic form;

2. Models of building energy consumption scenarios in a probabilistic form;
3. Models of the water management system;

4. Models of operating regimes of HPP and HPP cascades;

5. Models of the energy system.

Each block includes several models (simulation, optimization, and multi-criteria) that solve various problems, given the uncertainty of water inflows into 
reservoirs, the amplitude and frequency of temperature anomalies in the studied regions, the requirements of water users, the amount of electricity consumption, forced and planned repairs of electrical equipment and electrical networks, constraints on maximum transfer capability of individual sections of the power grid in controlled cutsets, other factors, and limitations.

Based on probabilistic long-term scenarios of water inflows into reservoirs and expected temperature conditions, the proposed approach provides optimal balanced operation of water and energy systems while ensuring reliability and uninterrupted energy and water supply to consumers at any considered time interval.

\subsection{Models for building long-term prognostic scenarios of water inflow into reservoirs}

In the context of global and regional climate changes, the use of hydro-meteorological statistics alone becomes ineffective for forecast estimates of water content and temperatures. Given the significant advancements in the creation and development of global climate models over the past decades, it seems appropriate to use them for long-term estimates of water availability and other hydro-meteorological indices. One of such models is the global climate model CFS-2 (Climate Forecast System) [19]. The results obtained with this model are updated daily in the form of ensemble forecasts of the state of the atmosphere and ocean with a time interval ranging from several hours to 10 months for the entire globe. The ensemble approach used in the model allows the formation of probabilistic estimates of the state of the atmosphere for the long term.

To monitor, accumulate and process the results of modeling, we developed special components that can quickly generate long-term estimates of precipitation, temperature, pressure, and geopotential in the river basins of the studied energy system. Figure 2, for example, illustrates the forecast maps for analyzing the climatic situation in the basins of Lake Baikal and the Angara reservoirs for the summer period of 2020, as of January 2020.

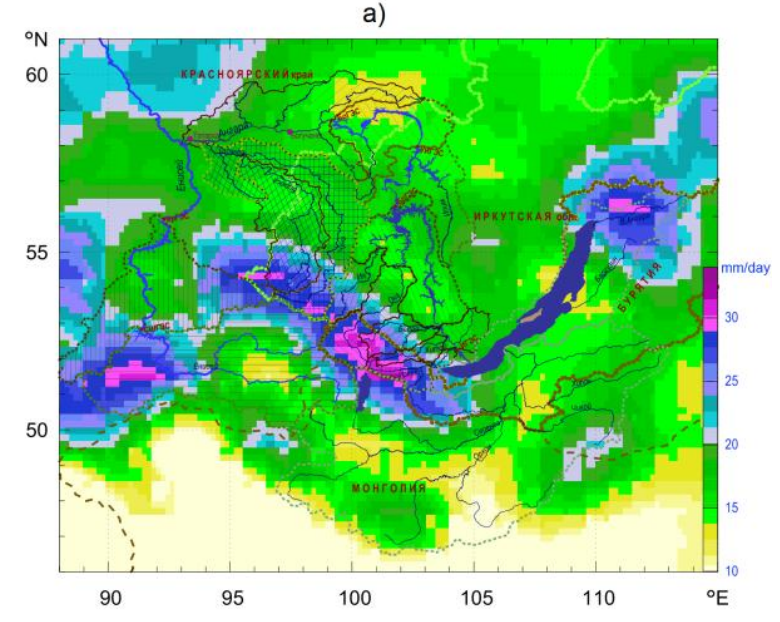

b)

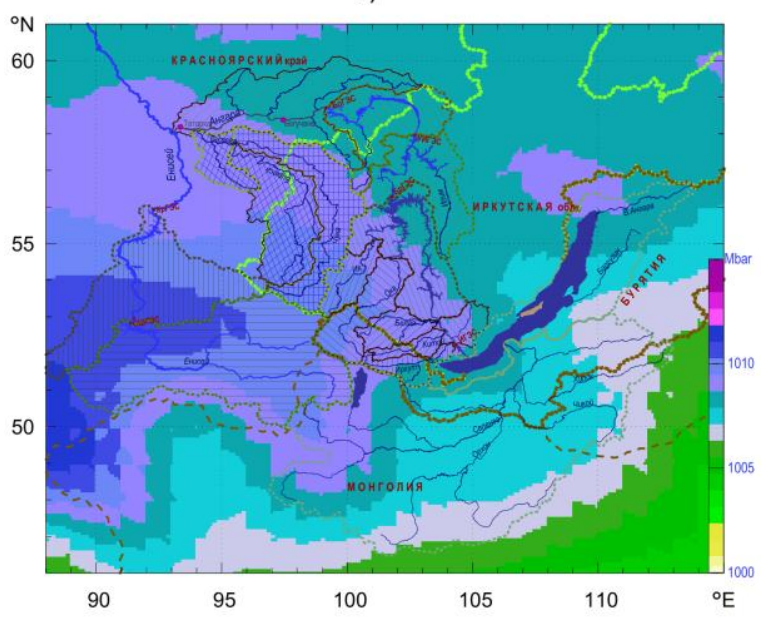

c)

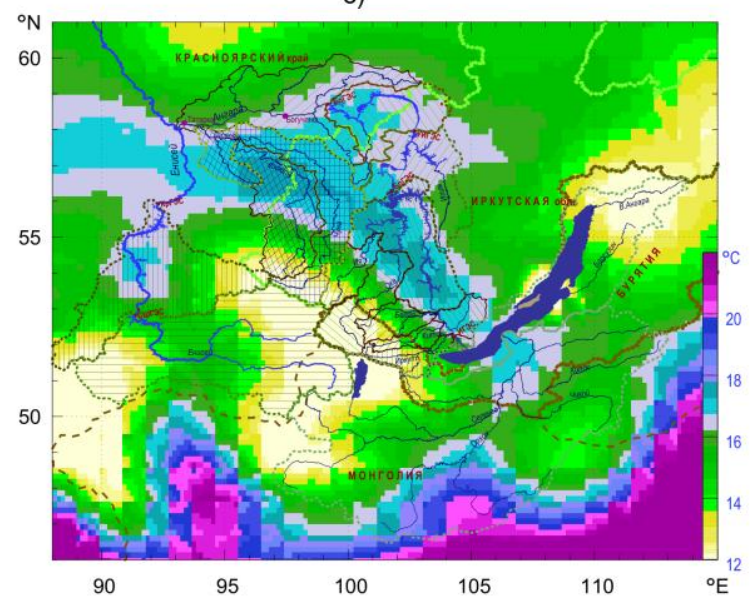

d)

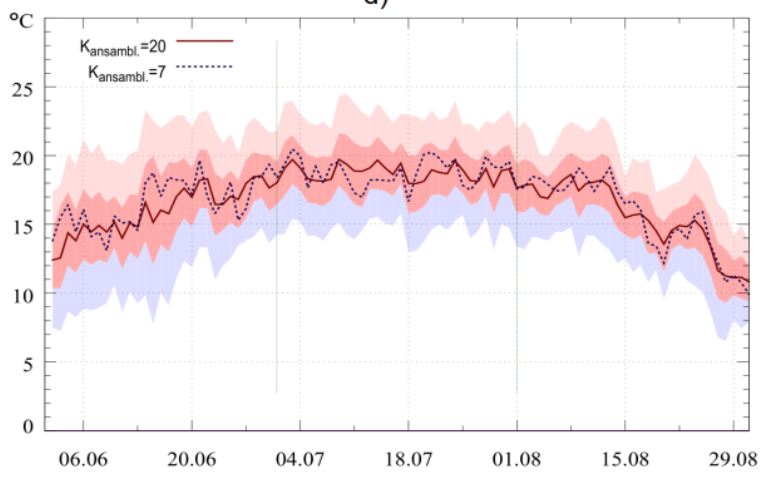

Fig. 2. An example of maps of prognostic indicators (average for the summer period of 2020): a) precipitation intensities; b) surface pressure at sea level; c) temperature conditions; d) dynamics of changes in surface temperatures in the area of the city of Irkutsk

Based on the processing of ensemble forecasts of meteorological indicators in the considered region, one determines analog years that are closest in atmospheric state characteristics. The found analogs are used to form estimates of inflows into the cascade reservoirs in the form of ranges of probability distributions. The period from April to October is the most important for planning the operating conditions of the water management system of Siberia. The final ensemble forecasts are generated by an automated procedure, which cuts off the 
unlikely events, processes regression relationships and relations between meteorological indices and river flows, and refines their boundaries based on expert estimates generated by other models $[20,21]$. Temperature conditions are formed as anomalies of their spatial distributions. In Siberia, the temperature conditions are essential for planning the operation of the energy system in the winter heating period (from November to March), when possible generation and consumption of thermal energy varies widely [22-24].

\subsection{Models for building long-term scenarios of electricity consumption in the energy system}

Electricity consumption in the energy system is probabilistic in nature. It is characterized by daily, seasonal, and interannual variability associated with the intensity of the consumer load, temperature conditions in the served territories, which significantly affects the heat output, and also with a possible connection of additional planned and unplanned consumers.

Models for building long-term scenarios of electricity consumption are based on the accumulated statistical data for various temperature conditions, which make it possible to form regression relationships, with a focus on periods of maximum and minimum consumption in daily and seasonal resolutions. Available planned indices of demand for electricity and power, commissioning of new large electricity consumers are also used.

\subsection{Water System Models}

The system includes hydrological models based on water balance equations; hydraulic models for estimating the travel time of the water flow to different points of the river network; water management models to take into account the constraints of all water users and water consumers, as well as environmental and social constraints. For example, a hydrological model of a linear cascade or its part can be represented as:

$$
\begin{aligned}
& \frac{d V_{i}}{d t}=P_{i}(t)-Q_{i}(t)+\bar{Q}_{i-1}(t)-\Lambda_{i}(t), \\
& Q_{B}(t)=Q_{4}(t)+P_{B}(t), Q_{T}(t)=Q_{B}(t)+P_{T}(t), \\
& Q_{0}(t)=0, \\
& \Lambda_{i}(t)=R_{i}^{i s p}(t)+R_{i}^{f i l t r}(t)+R_{i}^{p z}(t)+R_{i}^{t r}(t), \\
& t \in\left[t_{0}, T\right], i=\overline{1, N},
\end{aligned}
$$

where $i$ - HPP index; $t$ - time within a set interval $\left[t_{0}, T\right] ; V_{i}(t)$ - water volume in the reservoir; $P_{i}(t)-$ lateral (full) water inflow into reservoir; $Q_{i}(t)$ - flow rate through the HPP; $\bar{Q}_{i-1}(t)$ - flow rate of water of the upper stage of the HPP cascade, given water travel time; $Q_{B}, Q_{T}, P_{B}, P_{T}$ - flow rates and lateral inflows in the areas below the last stage, where the river levels are monitored; $\Lambda_{i}(t)$ - function of additional effect of evaporation from the reservoir surface $R_{i}^{i s p}(t)$, filtering through dam $R_{i}^{\text {filtr }}(t)$, changes in the underground component $R_{i}^{p z}(t)$, variations in the volume of water area between reservoir and upper stage of HPP. The values of functions $\Lambda_{i}(t)$, except for evaporation, can be neglected in practical calculations. This is due to the complexity of the estimates of these indices and their relatively small impact on the total inflow. The evaporation function plays a significant role in dry periods on Lake Baikal, therefore, its indices are included in the final available inflow to the Irkutsk reservoir.

For the specified time interval $\tau$ equation (1) can be approximately written in the finite difference form:

$$
\begin{aligned}
& \Delta V_{i}^{\tau}(t)=\left[Q_{i-1}^{\tau}(t)+P_{i}^{\tau}(t)-Q_{i}^{\tau}(t)-R_{i}^{i s p}(t)\right] \cdot \tau, \\
& Q_{0}^{\tau}(t)=0, i=\overline{1, N} .
\end{aligned}
$$

Constraints for upstream reach: $H_{\mathrm{i}}^{U M O} \leq H_{i}(t) \leq H_{i}^{N P U}$ - for normal water content, $H_{i}^{U M O-} \leq H_{i}(t) \leq H_{i}^{F P U}$ - for extreme water content.

Constraints on flow rates:

$$
q_{i}^{\text {turb }}(t, j) \leq q_{i}^{\max }\left(h_{i}\right), \quad q_{i}^{\text {hol }}(t, k) \leq q_{i}^{\text {hol, } \max }\left(h_{i}\right) .
$$

Statistical functions:

$$
\begin{aligned}
& z_{i}=\varphi_{i}^{z}\left(Q_{i}, H_{i+1}^{v b}\right), E_{i}^{\max }=\varphi_{i}^{E}\left(Q_{i}, h_{i}\right), \\
& H_{i}=\psi_{i}\left(V_{i}\right), V_{i}=\psi_{i}^{-1}\left(H_{i}\right),
\end{aligned}
$$

where $\Delta V_{i}^{\tau}, P_{i}^{\tau}, Q_{i}^{\tau}, R_{i}^{\tau}$ are average values of the functions on the time interval.

Functions of water inflow $P_{i}^{\tau}(t)$ have large interannual and seasonal variations, which affects greatly both the water system and the energy system.

The hydraulic models of steady-state flow can provide an estimate of the average flow at the point $\mathrm{X}$, located at a distance from the initial one:

$$
Q^{X}=1 / T \cdot\left[Q^{-} \Delta t+Q^{+}(T-\Delta t)+q^{-} \tau+q^{+}(T-\tau)\right],
$$

where $Q^{-}, Q^{+}$are flow rates at the initial crosssection for the previous and current ten days; $q^{-}, q^{+}-$ analogous lateral average ten-day inflows in the area; $\Delta t, \tau$ - travel time of the main and lateral inflows to point $X ; T$-duration of ten days (c).

The reservoir operating curves intended to manage the operating conditions at levels and given scenarios of water inflows into the reservoirs are used to make forecast scenarios of flow rates through HPP. The range of limiting flows goes to the block of models of HPP regime management.

In addition to feasible flow rates through HPP according to the requirements of water users and water consumers, the models of water management system determine periods and assess probable water management, environmental, and social risks.

\subsection{Models of operating conditions of HPP and HPP cascades}

Power and electricity output at individual HPP varies widely, which is related to seasonal and interannual unevenness of water inflows into reservoirs and operation of the energy system at different time intervals according to load curves. For the HPP cascade, as a rule, 
the interannual unevenness of the inflows decreases, except for cases of global low-water and high-water periods, covering large river basins.

Modeling the operating regimes of HPP according to the generated scenarios of water inflows into reservoirs solves the following problems:

1. Specification of water flow rates by reservoir operating curves:

$$
Q_{t}^{g}=D^{g}\left(H_{t}^{g}, P_{t}^{g}, \theta^{g}\right),
$$

where $D^{g}$ - reservoir operating curve of HPP with index $g ; \theta^{g}$ - additional vector of the reservoir operating curve parameters; $Q_{t}^{g}, H_{t}^{g}, P_{t}^{g}$ - flow rate, the upstream reach level, inflow into the reservoir at time $t$. With the sufficiently long hydrological statistics available, the reservoir operating curves allow building efficient operating conditions for any intra-annual and intramonth period for a given total inflow of water into the reservoir and the level of its upstream reach. The reservoir operating curves can be effectively used in simulation models for various studies. In the case of significant changes in hydrological characteristics compared to those observed previously, the effectiveness of their use in the calculation of flow rates decreases.

To determine the effective operating regimes of HPP to meet the set constraints on flow rates generated in the block of the water management system in the form of $Q_{i}^{\min }(t) \leq Q_{i}(t) \leq Q_{i}^{\max }(t), i=\overline{1, N}$, the ranges of flow rates for each time interval (a day, ten days, a month, a quarter) are specified using the reservoir operating curves given planned repairs.

2. Determination of flow rates for cascade management according to the criterion of maximization of electricity output from the entire cascade:

$$
\sum_{(t)} C_{t} \sum_{(g)}\left(E_{t}^{g}-E_{t}^{h o l}\right) \rightarrow \max
$$

with a set of constraints on flow rates, levels of upstream reach, power related to the type of loading the hydro units:

$$
\begin{aligned}
& Q_{t, \text { min }}^{g} \leq Q_{t}^{g} \leq Q_{t, \text { max }}^{g} \\
& H_{\text {min }}^{g} \leq H_{t}^{g} \leq H_{\text {max }}^{t} \\
& W_{\text {min }}^{g} \leq W_{t}^{g} \leq W_{\text {max }}^{t}
\end{aligned}
$$

Additional constraints are all water management constraints.

The optimization criterion may also be the maximum firm power of HPP in the winter period or for the entire water management year.

The output of this block is the feasible (possible) ranges of electricity output for each HPP, the entire HPP cascade, and indicators of used capacity in different periods.

For energy systems with a high share of hydropower plants, the main risks of operation management in the summer period are idle discharges through spillway structures of hydro systems, and an increased electricity output, when there is no corresponding demand and (or) it is impossible to fully use (transmit) it. The main risk in winter is the impossibility of providing firm power from HPP.

\subsection{Model of energy system}

Depending on the tasks being solved, which may include increasing the reliability and resilience of energy systems, increasing the economic efficiency of energy systems, assessing and minimizing various risks, preventing or reducing possible damage, rational planning of repairs, building up fuel reserves at Thermal Power Plants (TPP) and others, various formulations of the power system model are possible. In this case, the modeling of energy systems is reduced to solving the optimization problem, where the criterion for the efficiency of the energy system is reflected in the objective function, and the restrictions consist of balance restrictions and restrictions on variables. The main input parameters for the operation of the power system model block are: long-term energy consumption scenarios in annual, seasonal, monthly, weekly, daily and hourly sections; permissible operating modes of hydroelectric power stations taking into account all water management and energy restrictions, repair schedules for electrical equipment and electrical networks

One of the criterion of electricity generation cost minimization, the input data are flow characteristics of generating plants, the required levels of consumer loads in the territorial energy zones, transfer capabilities of tie lines, auxiliary power consumption, and factors of power losses in the transmission lines of the energy system. It is necessary to determine the optimal load of power plants given some technical limitations on the operation of the energy system and its facilities, and the potential surplus (shortage) of generating capacity.

The problem of planning the operation of the energy system for a period of up to 1 year is solved, the power balance is optimized for each hour of the calculation period.

Mathematically, the problem of optimizing the energy system operation is formulated as follows:

$$
\sum_{t} \sum_{i} \sum_{k} C_{t, i, k} \rightarrow \min
$$

subject to balance constraints:

$$
P_{t, i}-W_{t, i}+\sum_{(j)}\left(1-Z_{t, j i} \chi_{j i}\right) Z_{t, j i}-\sum_{(j)} Z_{t, i j}=0
$$

also variable constraints:

$$
\begin{aligned}
& \underline{P}_{t, i} \leq P_{t, i} \leq \bar{P}_{t, i}, \\
& P_{t, i}=\sum_{(n)} P_{t, i, n}+\sum_{(k)} P_{t, i, k}, \\
& C_{t, i, k}=\alpha_{i, k}+\beta_{i, k} P_{t, i, k}+1 / 2 \gamma_{i, k} P_{t, i, k}, \\
& 0 \leq Z_{t, i j} \leq \bar{Z}_{t, i j}, 0 \leq Z_{t, j i} \leq \bar{Z}_{t, j i}, \\
& t=1, \mathrm{~K}, T, i=1, \mathrm{~K}, I, k=1, \mathrm{~K}, K, \\
& n=1, \mathrm{~K}, N, j=1, \mathrm{~K}, J,
\end{aligned}
$$

where $C_{t, i, k}$ - the cost of generating electricity per hour $t$ at the $k$-th TPP in energy zone $i ; P_{t, i}-$ generated power per hour $t$ in energy zone $i ; W_{t, i}-$ power consumption per hour $t$ in energy zone $i ; Z_{t, j i}$ - power flow over the connection between energy zones $j$ and $i$ per hour $t ; \chi_{j i}$ - power loss coefficient; $Z_{t, i j}-$ power 
flow over the connection between energy zones $i$ and $j$ per hour $t ; \underline{P}_{t, i}$ - the total value of the minimum load of power plants per hour $t$ in the energy zone $i ; \bar{P}_{t, i}-$ the total value of the maximum load of power plants per hour $t$ in the energy zone $i ; P_{t, i, n}$ - loading of the $n$-th HPP per hour $t$ in the energy zone $i ; P_{t, i, k}$ - loading of the $k$-th TPP per hour $t$ in the energy zone $i ; \alpha_{i, k}, \beta_{i, k}$, $\gamma_{i, k}$ - the coefficients forming the flow characteristic of the $k$-th TPP in the energy zone $i ; T$ - the number of hours of the calculating period; $I$ - number of energy zones; $K$ - number of thermal power plants; $N$ - number of hydropower plants; $J$ - number of energy zones.

Problem (11)-(13) is solved by nonlinear programming methods. $P_{t, i, n}$ is determined when modeling the regimes of HPP and their cascades, while $P_{t, i, n}$ can have a constant value or be in the range $\underline{P}_{t, i, n} \leq P_{t, i} \leq \bar{P}_{t, i, n}$, where $\underline{P}_{t, i, n}$ is the minimum load of the $n$-th HPP at hour $t$ in the energy zone $i ; \bar{P}_{t, i, n}$ is the value of the maximum load of the $n$-th HPP per hour $t$ in the energy zone $i$.

After solving problem (11)-(13), an analysis is made of the results and modes in which no solution was found for various scenarios of water inflows and temperature conditions. As a result of several iterations according to model calculations with $P_{t, i, n}$ adjustment, the most optimal power system operating modes are selected according to the accepted criterion and electric power generation by the HPP of the power system.

\section{Conclusion}

The system of models presented in this paper allows studying the issues related to the management of HPP cascade operation within the water management and energy systems. It also enables us to formulate proposals for optimal long-term operating conditions, increase in stability, reliability, and efficiency of these systems while planning and managing them.

Long-term scenarios of changes in hydrometeorological indices (water inflows into reservoirs, temperatures, precipitation), obtained using the processed data from global climate models, provide an opportunity to assess in advance the probable risks of the impact of stochastic factors on the operation of the water and energy systems with a large share of HPP.

\section{Acknowledgements}

The research was carried out under State Assignment III.17.5.1 (reg. number AAAA-A17-117030310451-0) of the Fundamental Research of Siberian Branch of the Russian Academy of Sciences.

\section{References}

1. T.A. Filippova. Optimization of Power Flows of the Hydropower Units at Hydroelectric Plants (in Russian), Moscow, Energy, 208 p. (1975)

2. A.E. Asarin, K.I. Bestuzheva. Water and Energy Calculations (in Russian), Moscow, Energoatomizdat, 223 p. (1986)

3. E.V. Tsvetkov, T.M. Alyabysheva, L.G. Parfenov. Optimal Operating Conditions of Hydroelectric Plants in Electric Power Systems (in Russian). Moscow, Energoatomizdat, 304 p. (1984)

4. J. Keppo. Optimality with Hydropower System. Power Engineering Review, IEEE, 22, No. 6, 57 p. (2002)

5. M. Pereira, N. Campodonico, R. Kelman. Longterm Hydro Scheduling based on Stochastic Models, Proceeding of EPSOM Conference, Zurich, 22 p. (1998)

6. P.O. Pineau, L. Tranchecoste, and Y.VegaCardenas. Hydropower Royalties: A Comparative Analysis of Major Producing Countries (China, Brazil, Canada, and the United States), Water, 9, 287 (2017); DOI: 10.3390/w9040287

7. T.H. Bakken, A. Killingtveit, and K. Alfredsen. The Water Footprint of Hydropower Production - State of the Art and Methodological Challenges, 1, (2017); DOI: $10.1002 /$ gch2.201600018

8. T.H. Bakken, A. Killingtveit, K. Engeland, K. Alfredsen, and A. Harby. Water Consumption from Hydropower Plants - Review of Published Estimates and an Assessment of the Concept. Hydrol. Earth Syst. Sci., 17, pp. 3983-4000 (2013); DOI: 10.5194/hess-17-3983-2013

9. K. Bakker and C. Cook. Water Governance in Canada: Innovation and Fragmentation. Water Resources Development, 27, No. 2, pp. 275-289 (2011)

10. M. Ljungberg. Energy and hydrology modeling of hydropower in Eastern Canada. Water Resources Engineering, TVVR-15/5001, 79 p. (2015)

11. V.S. Dias, M.P. Luz, G.M. Medero, and D.T.F. Nascimento. An Overview of Hydropower Reservoirs in Brazil: Current Situation, Future Perspectives, and Impacts of Climate Change. Water, 10, 592 (2018); DOI: 10.3390/w10050592

12. Y. Jiang, F.K. S. Chan, J. Holden, Y. Zhao, and D. Guan. China's Water Management - Challenges and Solutions. Environmental Engineering and Management Journal, 12, No. 7, pp. 1311-1321 (2013)

13. Z. Feng, W. Niu, C. Cheng, X. Wu. Optimization of large-scale hydropower system peak operation with hybrid dynamic programming and domain knowledge, journal of Cleaner Production, pp. 390402 (2018); DOI: 10.1016/j.jclepro.2017.09.257

14. Specific features of Russia's hydropower industry operation under changing external conditions (on the example of the Volga-Kama cascade of hydropower plants), ed. by prof. A.Yu. Alexandrovsky and corr. 
member of RAS V.V. Klimenko (in Russian), Moscow, PH Energy, 169 p. (2016)

15. Water and energy regimes of hydroelectric plants in the context of climate change, ed. by Yu.S. Vasiliev (in Russian), St. Petersburg, Polytechnic University, 274 p. (2017)

16. S.E. Bednaruk, Yu.G. Motovilov. Information support technology for the reservoir cascade management (in Russian), Hydraulic engineering construction, No. 7, pp. 22-35 (2017)

17. E.N. Osipchuk, N.V. Abasov. Metamodelling technology for the study of HPP operating conditions (in Russian), proceedings of XVIII Baikal All-Russian conference "Information and mathematical technologies in science and management", 3, Irkutsk, ISEM SB RAS, pp. 274$280(2013)$

18. N.V. Abasov, V.M. Nikitin, E.N. Osipchuk. A System Of Models To Study Long-Term Operation Of Hydropower Plants In The Angara Cascade, Energy Systems Research, 2, No. 2(6), pp. 5-18 (2019)

19. The NCEP Climate Forecast System, available at http://cfs.ncep.noaa.gov (accessed on 20.07.2020)

20. T.V. Berejnykh, N.V. Abasov. The increasing role of long-term forecasting of natural factors in energy system management, International Journal of Global Energy Issues, 20, Issue 4, pp. 353-363 (2003); DOI: 10.1504/IJGEI.2003.004408

21. N.V. Abasov. The GeoGIPSAR system of long-term forecasting and analysis of environment-related factors of the energy industry (in Russian), Proceedings of the International Meeting of APN (MAIRS/NEESP/SIRS) Extreme manifestations of global climate change in North Asia, Enviromis2012, pp. 63-66 (2012)

22. Wenyuan Li. Probabilistic Transmission System Planning, Wiley-IEEE Press, 376 p. (2011)

23. Heejung Park, Ross Baldick, David P. Morton. A Stochastic Transmission Planning Model With Dependent Load and Wind Forecasts, IEEE Transactions on Power Systems, 30, issue 6, pp. 3003-3011 (2015)

24. Roy Billinton, Dange Huang. Effects of Load Forecast Uncertainty on Bulk Electric System Reliability Evaluation, IEEE Transactions on Power Systems, 23, issue 2, pp. 418-425 (2008) 